\title{
Making the Economic Value Proposition for Pharmacist Comprehensive Medication Management (CMM) in Primary Care: A Conceptual Framework
}

Kristen Tripicchio, PharmD Candidate, ${ }^{1}$ Sachiko Ozawa, PhD; ${ }^{1,2}$ Benjamin Urick, PharmD, PhD; ${ }^{1}$ and Jon Easter, BSPharm, Director for the Center for Medication Optimization. ${ }^{1}$

${ }^{1}$ Division of Practice Advancement and Clinical Education, University of North Carolina Eshelman School of Pharmacy, Chapel Hill, NC, 27516.

${ }^{2}$ Department of Maternal and Child Health, University of North Carolina Gillings School of Global Public Health, Chapel Hill, NC, 27516. 


\section{ABSTRACT}

Introduction: Comprehensive medication management (CMM) is a patient care process provided by clinical pharmacists in primary care settings that ensures optimal use of medications with timely followup. Despite widespread evidence that shows CMM improves clinical and medication-related outcomes, pharmacist CMM services often fail to be adopted into U.S. primary care settings.

Objective: This study presents a conceptual framework linking outcomes of pharmacist-delivered CMM services in primary care settings to financial benefits for both primary care practices investing and implementing $\mathrm{CMM}$ and health plans providing coverage of CMM services.

Methods: A narrative literature review was performed in PubMed and the gray literature to identify financing opportunities that justify the coverage of $\mathrm{CMM}$ by third party health plan administrators or the implementation of CMM by primary care practices. Financing elements that could be impacted by pharmacist-led CMM outcomes, namely higher achievement of medication-related quality measures and reduction of total costs of care, were recorded and utilized to develop the conceptual framework.

Results: The framework suggests that CMM may provide economic benefits to health plans and care practices by (1) increasing market competitiveness through higher quality ratings, accreditation recognition, lower premiums and plan bids, and increased patient satisfaction; and (2) increasing revenue through quality bonus payments, shared savings agreements, and fee-for-service reimbursement.

Conclusions: The alignment of economic benefits that can be realized through CMM advances a strong value proposition for greater adoption of CMM in the United States' primary care system. Future application of this study's conceptual framework could include use as an advocacy tool amongst pharmacy associations or as a tool for individuals seeking buy-in for CMM services. 


\section{INTRODUCTION}

The misuse, underuse, and overuse of medications is one of the largest preventable factors contributing to lower health care quality in the United States. While prescription drug spending in the U.S. is estimated to be $\$ 330$ billion of the $\$ 3.3$ trillion in total health expenditures, costs resulting from non-optimized medication therapy have been estimated to exceed $\$ 500$ billion, implying that for every dollar spent on medications an additional dollar is needed to address inappropriate medication use. ${ }^{1,2}$ One solution for reducing unnecessary medication-related spending is comprehensive medication management (CMM), a clinical service delivered by pharmacists using an integrated care team approach. The CMM patient care process ensures that each patient's medications are evaluated to determine appropriateness, effectiveness, safety, adherence, and accessibility resulting in an individualized care plan with timely follow-up. ${ }^{3}$

Pharmacist-led CMM has consistently demonstrated positive clinical outcomes most often achieved through identification and resolution of medication therapy problems (MTPs). On average, studies of CMM services have shown identification and resolution of 4.5 MTPs per patient with the most frequent type of MTP identified including need for additional medication therapy or suboptimal dosing of an existing therapy. ${ }^{4-13}$ Examples of relevant CMM interventions include addition of indicated statins or asthma inhaler therapy, increasing beta blocker doses for heart failure patients, or discontinuing high risk medications in elderly patients.

In terms of diabetes management, studies have shown that CMM leads to significant reductions in hemoglobin A1c compared to control arms with estimated treatment differences ranging from $-0.4 \%$ to $-1.7 \% .{ }^{14-18}$ Moreover, several studies have demonstrated CMM's positive impact on all-or-none diabetes composite scores that involve achievement of several important treatment goals including smoking cessation, daily aspirin use, and meeting A1c, low density lipoprotein (LDL), and blood pressure goals. ${ }^{5-7,15}$ Some studies of well-adopted CMM services have even demonstrated a positive impact on lowering patient total cost of care by decreasing hospitalizations and other acute care utilization. ${ }^{8,13}$

Despite widespread evidence that shows CMM improves clinical and medication-related outcomes, pharmacist CMM services fail to be consistently adopted into U.S. primary care settings. ${ }^{19-}$ 21,22 CMM services are often funded through unsustainable means such as temporary grants, ${ }^{11,12,16,23}$ pilot programs, ${ }^{8}$ or limited fee-for-service billing reimbursement. ${ }^{5,6,9,10}$

Fortunately, the current healthcare policy landscape and value-based directive in the U.S. presents a favorable environment for the economic justification of pharmacist CMM services. In the new age of emphasis on value and the partial transfer of risk from payers to delivery systems, CMM has the potential to be financially viable. ${ }^{24,25}$ This study presents a theoretical framework to conceptualize how CMM-driven outcomes can financially benefit third party health plans and primary care delivery systems deliberating about whether to reimburse for or implement pharmacist CMM services.

\section{METHODS}

A critical review of the literature was performed to discern financing opportunities that justify the coverage of $\mathrm{CMM}$ by third party health plan administrators or the implementation of CMM by primary care practices.

In order to develop the narrative framework, a paradigm was applied that presumes pharmacist CMM services result in lower total costs of care through decreased acute care utilization and higher achievement of medication-related quality measures. Thus, financing elements that could be positively impacted by either lower total costs of care or higher achievement of quality measures were assumed to contribute to the economic justification for CMM. This paradigm is supported by the literature and continues to be evaluated as the value of clinical pharmacy services gains national attention. 
The literature review focused on financing elements between U.S. healthcare financers (individuals, employers, and government), third party health plan administrators, and primary care delivery systems. Specifically, the scope of the framework was limited to health insurance through Medicare, Medicaid, fully or self-insured employers, and the exchange marketplace as these make up the bulk of health financing in the United States. ${ }^{26-28}$ Financing of primary care practices inclusive of physicians organized into Accountable Care Organizations (ACOs) or Patient-Centered Medical Homes (PCMHs) was also evaluated.

Literature searches were conducted in PubMed and the gray literature through use of key terms and synonyms that reflected financing models between the chosen stakeholders (e.g. Medicare Advantage financing, ACO Medicare financing, etc). Titles and abstracts were scanned to identify articles that discussed relevant revenue models. For financing elements where more detailed information was available (e.g. Medicare Advantage Stars quality bonus payments), secondary searches were performed to understand contract specifics such as relevant quality metrics or risk-adjustment factors. Financing elements that could be impacted by the stated paradigm were recorded and utilized to develop the conceptual framework.

\section{RESULTS}

\section{CMM Value Proposition within the U.S. Healthcare System: A Conceptual Framework}

This framework presents the economic value proposition for CMM in primary care settings (Figure 1). Within the framework are three key categories of stakeholders, including financers of healthcare (i.e. employers, individuals, and the government), third party health plans that facilitate contracts and health benefits on behalf of financers, and primary care practices where CMM services may be implemented. Individuals traditionally receive health insurance through their employer, direct purchase facilitated by an exchange, or eligibility for government programs. Employers may provide healthcare benefits to their employees by contracting with a third-party payer (fully-insured) or by contracting directly with healthcare systems (self-insured). ${ }^{26}$ Similarly, Medicare and Medicaid programs may be facilitated through contracts with third party health plans (i.e. Medicare Part C and Medicaid managed care) or direct contracts with healthcare delivery systems (i.e. traditional Medicare and non-managed Medicaid). ${ }^{27,28}$

The following sections will narrate how clinical and economic outcomes from CMM services can benefit third party health plans and primary care delivery systems through various mechanisms elucidated from the literature review.

\section{CMM From a Third-Party Health Plan Perspective}

The primary lines of business for third party health plans include Medicare Advantage (MA) plans, state Medicaid managed care plans, and commercial plan contracts purchased by employers or individuals. CMM has the opportunity to benefit all lines of business through the following mechanisms: (1) increased market competitiveness, (2) achievement of quality bonus payments, and (3) increased revenue from capitated shared savings contracts.

Coverage of CMM services may increase health plan market competitiveness by lowering health plan total medical costs, thus allowing plans to lower their bids within request for proposals (RFPs) to Medicare and Medicaid or offer lower premiums within the commercial market. ${ }^{29}$ In addition, CMM has the potential to increase market competitiveness by improving plan ratings. In the commercial market, the National Committee for Quality Assurance (NCQA) accredits health plans and assigns plan quality 
ratings, both of which are essential for winning contracts from large employers looking to provide a high-quality benefit package to their employees. ${ }^{30,31}$ NCQA ratings and accreditation are based on achievement of Healthcare Effectiveness Data Information Set (HEDIS) quality measures including process (e.g. statin therapy in cardiovascular disease), outcome (e.g. controlled A1c, reduction in hospitalizations), and patient experience measures. ${ }^{31,32}$ Over $30 \%$ of HEDIS quality measures are related to medications or medication-related treatment outcomes that are either a result of CMM MTP resolution (e.g. addition of an indicated statin) or that serve as a goal of CMM care (e.g. controlled blood pressure). ${ }^{32}$ Similarly, in the Medicare market, Stars Ratings serve as a way to rank a high-quality plan. Of the 48 Stars Measures for 2018, 15 (31\%) are either related to medication management (e.g. diabetes medication adherence, achievement of blood pressure control) or outcomes positively influenced by CMM such as hospital readmissions (see Table 1). ${ }^{33}$ Of note, $66 \%$ of Medicare Advantage enrollees in 2017 were enrolled in a plan with a Star Rating of 4 or more Stars on the 5-star scale, indicating the impact of high quality ranking on member enrollment. ${ }^{28}$

$\mathrm{CMM}$ can directly influence plan revenue through achievement of quality bonus payments. In the Medicare Advantage program, a quality bonus incentive payment of $5 \%$ is given to those plans who achieve a Star rating of 4 or higher. ${ }^{34}$ Quality bonus payments are also present in some state Medicaid programs such as New York, where plans may receive bonuses upon high quality measure performance. ${ }^{35}$

Lastly, through lowering cost of care and higher achievement of quality measures, CMM can benefit plans engaged in capitated shared savings contracts such as the Medicare Advantage payment system. In the MA market, plans submit bids to CMS that estimate the cost to cover an average beneficiary. Plans who submit bids lower than a CMS calculated benchmark rate have the opportunity to gain a portion of the "savings" (difference between the benchmark and plan bid). ${ }^{29}$ Lowering the total cost of care of a plan population through mediation management can help plans submit lower bids and recoup a larger magnitude of savings. In addition, the proportion of savings that is repaid to Medicare Advantage plans, also known as the MA rebate, is dictated by Star Ratings which may be impacted by CMM. Plans with 4.5 stars or higher receive a $70 \%$ rebate, whereas plans with 3 stars or lower only receive a $50 \%$ rebate. ${ }^{34}$

\section{CMM From a Primary Care Practice Perspective}

Primary care practices may be primarily funded through traditional FFS reimbursement or organized into models where care coordination, quality, and cost-effectiveness are emphasized such as patientcentered medical homes (PCMHs) or accountable care organizations (ACOs). Depending on practice structure, $\mathrm{CMM}$ has the opportunity to benefit primary care practices through (1) increased market competitiveness, (2) traditional FFS payments, (3) quality bonus payments, and (4) shared savings.

Integrating CMM into primary care practices can increase market competitiveness with consumers and health plans. Consumers have demonstrated high patient satisfaction with $\mathrm{CMM}$ services and increased confidence in managing their disease. ${ }^{4,5,10,14}$ Practices may use patient anecdotes as a marketing tactic to increase volume. In addition, $\mathrm{CMM}$ can increase contract negotiating power with health plans. First, CMM can help improve quality metrics, patient satisfaction, and acute care utilization which provide important data that can be leveraged in insurer negotiations. Second, CMM implementation can help with requirements for ACO or medical home accreditation. For example, the NCQA PCMH Recognition process requires team-based care, delivery of guideline-directed care, medication education and counseling, establishment of person-centered care plans for high risk patients, and other requirements that can be addressed by the CMM care process. ${ }^{36}$ Not only does recognition as a medical home or ACO improve practice bargaining power and market competitiveness 
with health plans, but it can also increase practice revenue in the form of additional care management fees, quality bonuses, or shared savings. ${ }^{37,38}$

Pharmacist-delivered CMM services can also generate direct revenue from traditional FFS billing. Although FFS reimbursement is often insufficient to cover all costs associated with CMM, it can provide consistent supplemental revenue in addition to the other economic benefits discussed in this framework. Certain states such as Minnesota have recognized the value of pharmacist CMM services and have created a FFS reimbursement structure to recognize CMM care delivery. ${ }^{6}$ However, for the majority of practice settings, FFS billing for CMM can either be achieved directly though CPT code 99211 (\$20-\$30) or through physician "incident-to" billing for a variety of scenarios where reimbursement may range from $\$ 40$ to $\$ 226$ (see Table 2). ${ }^{39}$ These may include transitional care management (CPT 99495, 99496), annual wellness visits (G0438, G0439), or newer chronic care management codes (CPT 99490)..$^{40}$ Practices have the opportunity to be innovative with the CMM care process in order to receive maximal reimbursement. Two real-world examples of this include incorporating Annual Wellness Visits into the $\mathrm{CMM}_{\text {process }}{ }^{29}$ and $\mathrm{MD} /$ PharmD co-appointments that allow for physicians to see more patients daily and bill at a higher complexity. ${ }^{41}$

Practices can also achieve direct revenue through quality bonus incentive payments with commercial and government payers through the Merit-Based Incentive Payment System (MIPS) and pay-for-performance (P4P) models. ${ }^{42,43}$ Due to the recent Medicare physician reimbursement overhaul known as the Medicare Access and CHIP Reauthorization Act of 2015, primary care physicians will be engaged in upside-downside risk adjustments that can affect up to $9 \%$ of their Medicare reimbursement. ${ }^{43}$ These adjustments will be based on MIPS composite scores that incentivize quality, efficient resource utilization, practice quality improvement activities, and advancement of health information technology. Sixty percent of the MIPS score is made up of achievement of quality measures and resource utilization (i.e. cost of medical claims), which may be positively impacted by CMM interventions. ${ }^{43}$ Several MIPS quality measures are related to chronic medication management such as evidence-based therapy for heart failure, improvement in blood pressure, and optimal asthma control. ${ }^{44}$ In addition to Medicare's MIPS program, pay-for-performance models are another form of quality bonus payments. P4P arrangements vary widely and are ubiquitous across Medicare, Medicaid, and commercial contracts. ${ }^{42} \mathrm{P} 4 \mathrm{P}$ may be based on achievement of quality measures such as following guideline-based therapy or based on lowering acute care utilization, both of which can be supported by the CMM care model.

Lastly, through lowering acute care utilization, CMM has the potential to increase shared savings for practice settings where capitation is the primary reimbursement model such as in ACOs. As health systems take on more risk for attributed patient panels, investment in services that aim to manage chronic disease and prevent avoidable complications will be integral for driving revenue. ${ }^{45}$

\section{DISCUSSION}

This study presents a novel conceptual framework describing the economic value proposition for CMM in the U.S. healthcare system from a dual stakeholder perspective. The framework demonstrates aligned economic justification for CMM by both primary care practices investing and implementing $\mathrm{CMM}$ and health plans providing coverage of CMM services. Namely - depending on the patient mix, health plans and care practices may increase market competitiveness and increase revenue by adopting CMM.

Health plans and care practices alike can increase their market competitiveness through CMM services. Specifically, health plans have the opportunity to increase their competitiveness with Medicare, patients, and even employer groups through achieving lower RFP bids or premiums and achieving higher quality metrics that allow for higher plan ratings and NCQA accreditation. Similarly, care practices have the opportunity to remain competitive with contracted plans and patients. CMM 
services give care practices the marketing opportunity to tout high patient engagement, exceptional follow up, and disease management education to complex patients who often feel overwhelmed by the system. In addition, through greater achievement of quality metrics and adoption of the CMM care model, care practices may increase their negotiating power with contracted health plans.

CMM services can also directly and indirectly drive revenue for health plans and care practices through quality bonus payments, shared savings agreements, and fee-for-service reimbursement. Ultimately, the focal point of these revenue opportunities for health plans and care practices alike is centered around achievement of value-based quality measures as well as lowering total medical costs. Given that the sustainability of CMM implementation is based on these outcomes, practices and health plans should carefully consider quality measures they are held accountable for and create infrastructure to help improve those outcomes. Health plans should focus on using analytics to identify quality opportunities, improve communication with providers and care practices to address those gaps, and create practice-level incentives for quality measures that can help fund and sustain CMM services. Similarly, care practices should also invest in analytics to identify quality opportunities, educate providers and care team members on the importance of quality-based initiatives, and ensure their CMM care process is nimble in order to continuously address different gaps in care.

There are a few limitations to this study. First, publicly available information regarding commercial contract data between employers and health plans as well as health plans and care practices is very limited. Although the literature suggests that there is an increasing commercial focus on value and quality measures, the magnitude and specifics of this change remains unknown. Future studies could attempt to address this gap in the literature through surveys and focus groups of commercial payers.

Second, this framework is dependent on the stated paradigm that CMM services result in lower total costs of care and higher achievement of medication-related quality measures. There is a growing body of literature that supports this paradigm; however, it remains difficult to ascertain macro level changes such as an improvement in a plan's Star rating to a single intervention. In order to combat this, care practices should remain committed to tracking interventions and outcomes to continue generating evidence for $\mathrm{CMM}$ services. One innovation that may help with tracking outcomes is the introduction of SNOMED CT codes, a new coding system to record care interventions in electronic health record (EHR) systems. Adoption and consistent use of SNOMED CT codes as a way to track and monitor interventions made by pharmacists can present an opportunity to make a more convincing value argument for CMM. ${ }^{46}$

Finally, this framework is qualitative in nature, and thus the magnitude of the economic benefits of $\mathrm{CMM}$ are not evaluated here. Future studies could work to develop a flexible model that allows health plans and care practices to input their population mix (e.g. Medicare, Medicaid, commercial) and analyze how incorporation of CMM services could positively influence their bottom line.

Future application of this study's conceptual framework could include use as an advocacy tool amongst pharmacy associations or as a tool for individuals seeking buy-in for CMM services. Examples of this may include primary care practices looking to convince health plans to reimburse for CMM within their contracts or pharmacists looking to convince health system administrators to implement CMM services within additional clinics. In both of these scenarios, this conceptual framework can serve as a starting point for negotiations or business plan development.

\section{CONCLUSIONS}

This study presents a novel framework for justifying the adoption of pharmacist comprehensive medication management services in the United States. By improving healthcare quality measures and decreasing acute complications of chronic disease management, CMM can increase health plan and 
primary care practice market competitiveness and increase revenue. The broad alignment of benefits that can be realized across the healthcare system advances a strong value proposition for greater adoption of CMM in the United States' primary care system.

Acknowledgments: The authors would like to acknowledge Drs. Mary Roth McClurg and Carrie Blanchard for their support and research guidance of this project.

Funding Source: This research did not receive any specific grant from funding agencies in the public, commercial, or not-for-profit sectors.

Competing Interests: The authors have no competing interests to disclose. 


\section{REFERENCES}

1. National Health Expenditure Fact Sheet. Centers for Medicare \& Medicaid Services. https://www.cms.gov/ResearchStatistics-Data-and-Systems/Statistics-Trends-and-Reports/NationalHealthExpendData/NHE-Fact-Sheet.html. Published 2018. Accessed April 7, 2018.

2. Watanabe JH, McInnis T, Hirsch JD. Cost of Prescription Drug-Related Morbidity and Mortality. Ann Pharmacother. March 2018:106002801876515. doi:10.1177/1060028018765159.

3. Blanchard C, Livet M, Ward C, Sorge L, Sorensen TD, McClurg MR. The Active Implementation Frameworks: A roadmap for advancing implementation of Comprehensive Medication Management in Primary care. Res Social Adm Pharm. 2017;13(5):922-929. doi:10.1016/j.sapharm.2017.05.006.

4. Shimp LA, Kucukarslan SN, Elder J, et al. Employer-based patient-centered medication therapy management program: evidence and recommendations for future programs. J Am Pharm Assoc (2003). 2012;52(6):768-776. doi:10.1331/JAPhA.2012.11186.

5. Ramalho de Oliveira D, Brummel AR, Miller DB, et al. Medication Therapy Management: 10 Years of Experience in a Large Integrated Health Care System. J Manag Care Pharm. 2010;16(185):185-195. doi:10.18553/jmcp.2010.16.3.185.

6. Isetts BJ. Evaluating Effectiveness of the Minnesota Medication Therapy Management Care Program.; 2007. https://www.leg.state.mn.us/docs/2008/mandated/080113.pdf. Accessed March 2, 2018.

7. Isetts BJ, Brummel AR, de Oliveira DR, Moen DW. Managing drug-related morbidity and mortality in the patientcentered medical home. Med Care. 2012;50(11):997-1001. doi:10.1097/MLR.0b013e31826ecf9a.

8. Altavela JL, Jones MK, Ritter M. A prospective trial of a clinical pharmacy intervention in a primary care practice in a capitated payment system. J Manag Care Pharm. 2008;14(9):831-843. doi:10.18553/jmcp.2008.14.9.831.

9. Truong H-A, Groves CN, Congdon HB, Dang D-TT, Botchway R, Thomas J. Potential cost savings of medication therapy management in safety-net clinics. J Am Pharm Assoc (2003). 2015;55(3):269-272. doi:10.1331/JAPhA.2015.14062.

10. Woodall T, Landis SE, Galvin SL, Plaut T, McClurg MTR. Provision of annual wellness visits with comprehensive medication management by a clinical pharmacist practitioner. Am J Heal Pharm. 2017;74(4):218-223. doi:10.2146/ajhp150938.

11. Roth MT, Ivey JL, Esserman DA, et al. Individualized medication assessment and planning: Optimizing medication use in older adults in the primary care setting. Pharmacotherapy. 2013;33(8):787-797. doi:10.1002/phar.1274.

12. Smith M, Giuliano MR, Starkowski MP. In Connecticut: improving patient medication management in primary care. Health Aff (Millwood). 2011;30(4):646-654. doi:10.1377/hlthaff.2011.0002.

13. Isetts BJ, Schondelmeyer SW, Artz MB, et al. Clinical and economic outcomes of medication therapy management services: the Minnesota experience. J Am Pharm Assoc (2003). 2008;48(2):203-11; 3 p following 211. doi:10.1331/JAPhA.2008.07108.

14. Rothman RL, Malone R, Bryant B, et al. A randomized trial of a primary care-based disease management program to improve cardiovascular risk factors and glycated hemoglobin levels in patients with diabetes. Am J Med. 2005;118(3):276-284. doi:10.1016/j.amjmed.2004.09.017.

15. Brummel AR, Soliman AM, Carlson AM, de Oliveira DR. Optimal diabetes care outcomes following face-to-face medication therapy management services. Popul Health Manag. 2013;16(1):28-34. doi:10.1089/pop.2012.0023.

16. McAdam-Marx C, Dahal A, Jennings B, Singhal M, Gunning K. The effect of a diabetes collaborative care management program on clinical and economic outcomes in patients with type 2 diabetes. J Manag care Spec Pharm. 2015;21(6):452-468. doi:10.18553/jmcp.2015.21.6.452.

17. Chung N, Rascati K, Lopez D, Jokerst J, Garza A. Impact of a clinical pharmacy program on changes in hemoglobin A1c, diabetes-related hospitalizations, and diabetes-related emergency department visits for patients with diabetes in an underserved population. J Manag care Spec Pharm. 2014;20(9):914-919. doi:10.18553/jmcp.2014.20.9.914.

18. Ip EJ, Shah BM, Yu J, Chan J, Nguyen LT, Bhatt DC. Enhancing diabetes care by adding a pharmacist to the primary care team. Am J Health Syst Pharm. 2013;70(10):877-886. doi:10.2146/ajhp120238.

19. Viswanathan M, Kahwati LC, Golin CE, et al. Medication Therapy Management Interventions in Outpatient Settings. JAMA Intern Med. 2015;175(1):76. doi:10.1001/jamainternmed.2014.5841.

20. Chisholm-Burns MA, Kim Lee J, Spivey CA, et al. US Pharmacists' Effect as Team Members on Patient Care. Med Care. 2010;48(10):923-933. doi:10.1097/MLR.0b013e3181e57962.

21. Perlroth D, Marrufo G, Montesinos A, et al. Medication Therapy Management in a Chronically III Population: Final Report.; 2010. https://innovation.cms.gov/files/reports/mtm_final_report.pdf. Accessed April 8, 2018.

22. Evidence Supporting Enhanced Medication Therapy Management. https://innovation.cms.gov/Files/x/mtmevidencebase.pdf.

23. Romanelli RJ, Leahy A, Jukes T, Ishisaka DY. Pharmacist-led medication management program within a patientcentered medical home. Am J Health Syst Pharm. 2015;72(6):453-459. doi:10.2146/ajhp140487.

24. Smith M, Bates DW, Bodenheimer TS. Pharmacists Belong In Accountable Care Organizations And Integrated Care Teams. Health Aff. 2013;32(11):1963-1970. doi:10.1377/hlthaff.2013.0542. 
25. Brummel A, Lustig A, Westrich K, et al. Best practices: improving patient outcomes and costs in an ACO through comprehensive medication therapy management. J Manag care Spec Pharm. 2014;20(12):1152-1158.

26. Claxton G, Rae M, Long M, Damico A, Foster G, Whitmore H. Employer Health Benefits 2017 Annual Survey.; 2017. http://files.kff.org/attachment/Report-Employer-Health-Benefits-Annual-Survey-2017. Accessed April 5, 2018.

27. Barnett JC, Berchick ER. Health Insurance Coverage in the United States: 2016.; 2017. https://www.census.gov/library/publications/2017/demo/p60-260.html. Accessed February 23, 2018.

28. Jacobson G, Damico A, Neuman T. Medicare Advantage 2017 Spotlight: Enrollment Market Update.; 2017. https://www.kff.org/medicare/issue-brief/medicare-advantage-2017-spotlight-enrollment-market-update/. Accessed April 6, 2018.

29. Medicare Advantage Program Payment System.; 2016. http://www.medpac.gov/docs/default-source/paymentbasics/medpac_payment_basics_16_ma_final.pdf. Accessed April 6, 2018.

30. NCQA Health Insurance Plan Ratings 2018. http://www.ncqa.org/report-cards/health-plans/health-insurance-planratings/ncqa-health-insurance-plan-ratings-2018. Accessed April 6, 2018.

31. NCQA Health Insurance Plan Ratings Methodology, March 2018. www.ncqa.org. Published 2018. Accessed April 6, 2018.

32. HEDIS 2018 Measures: Summary Table of Measures, Product Lines, and Changes. http://www.ncqa.org/Portals/0/HEDISQM/HEDIS2018/HEDIS 2018 Measures.pdf?ver=2017-06-28-134644-370. Accessed April 6, 2018.

33. 2018 Part C \&amp; D Star Ratings Measures. https://www.cms.gov/Medicare/Prescription-DrugCoverage/PrescriptionDrugCovGenIn/Downloads/2018MeasureList.pdf. Published 2018. Accessed April 6, 2018.

34. Grzeskowiak D. Medicare Advantage Star Ratings: Basics and Best Practices.; 2017. http://www.milliman.com/uploadedFiles/insight/2017/medicare-advantage-star-ratings-best-practices.pdf. Accessed April 6, 2018.

35. Report to the Congress: The Evolution of Managed Care in Medicaid.; 2011. https://www.macpac.gov/wpcontent/uploads/2015/01/Payment_Policy_in_Medicaid_Managed_Care.pdf. Accessed April 6, 2018.

36. NCQA PCMH Standards and Guidelines.; 2017. http://store.ncqa.org/index.php/catalog/product/view/id/2776/s/2017pcmh-standards-and-guidelines-epub/. Accessed April 7, 2018.

37. Centers for Medicare and Medicaid Services. Comprehensive Primary Care Plus. https://innovation.cms.gov/initiatives/comprehensive-primary-care-plus. Published 2018. Accessed April 7, 2018.

38. Nielsen M, Langner B, Zema C, Hacker T, Grundy P. Benefits of Implementing the Primary Care Patient-Centered Medical Home: A Review of Cost \&amp; Quality Results.; 2012.

https://www.pcpcc.org/sites/default/files/media/benefits_of_implementing_the_primary_care_pcmh.pdf. Accessed March 23, 2018.

39. Physician Fee Schedule Search. Centers for Medicare and Medicaid Services. https://www.cms.gov/apps/physician-feeschedule/search/search-results. aspx?Y=0\&T=0\&HT=0\&CT=3\&H1=99495\&M=5. Accessed April 7, 2018.

40. Coffey J. Pharmacist Billing for Ambulatory Pharmacy Patient Care Services in a Physician- Based Clinic and Other NonHospital-Based Environments - FAQ. Am Soc Heal Syst Pharm. 2014.

41. Ulrich IP, Patel S, Gilmer B. Evaluation of a pharmacist-physician covisit model in a family medicine practice. J Am Pharm Assoc. November 2018. doi:10.1016/J.JAPH.2018.09.010.

42. Friedberg $\mathrm{M}$, Chen $\mathrm{P}$, White $\mathrm{C}$, Jung $\mathrm{O}$. Effects of health care payment models on physician practice in the United States. St Monica RAND. 2015. http://130.154.3.8/content/dam/rand/pubs/research_reports/RR800/RR869/RAND_RR869.pdf. Accessed February 2, 2017.

43. Centers for Medicare and Medicaid Services. The Merit-Based Incentive Payment System: MIPS Scoring Methodology Overview. https://www.cms.gov/Medicare/Quality-Initiatives-Patient-Assessment-Instruments/Value-BasedPrograms/MACRA-MIPS-and-APMs/MIPS-Scoring-Methodology-slide-deck.pdf. Accessed April 7, 2018.

44. MIPS Quality Measures. Centers for Medicare and Medicaid Services. https://qpp.cms.gov/mips/quality-measures. Accessed April 7, 2018.

45. Brummel A, Lustig A, Westrich $\mathrm{K}$, et al. Best practices: improving patient outcomes and costs in an ACO through comprehensive medication therapy management. J Manag care Spec Pharm. 2014;20(12):1152-1158.

46. SNOMED CT Codes: Making the Case for Pharmacy Services with Hard Data. American Society of Health-System Pharmacists. 2015.

\section{FIGURES AND TABLES}


Figure 1. Conceptual framework describing the economic value proposition for CMM within the U.S. healthcare system.

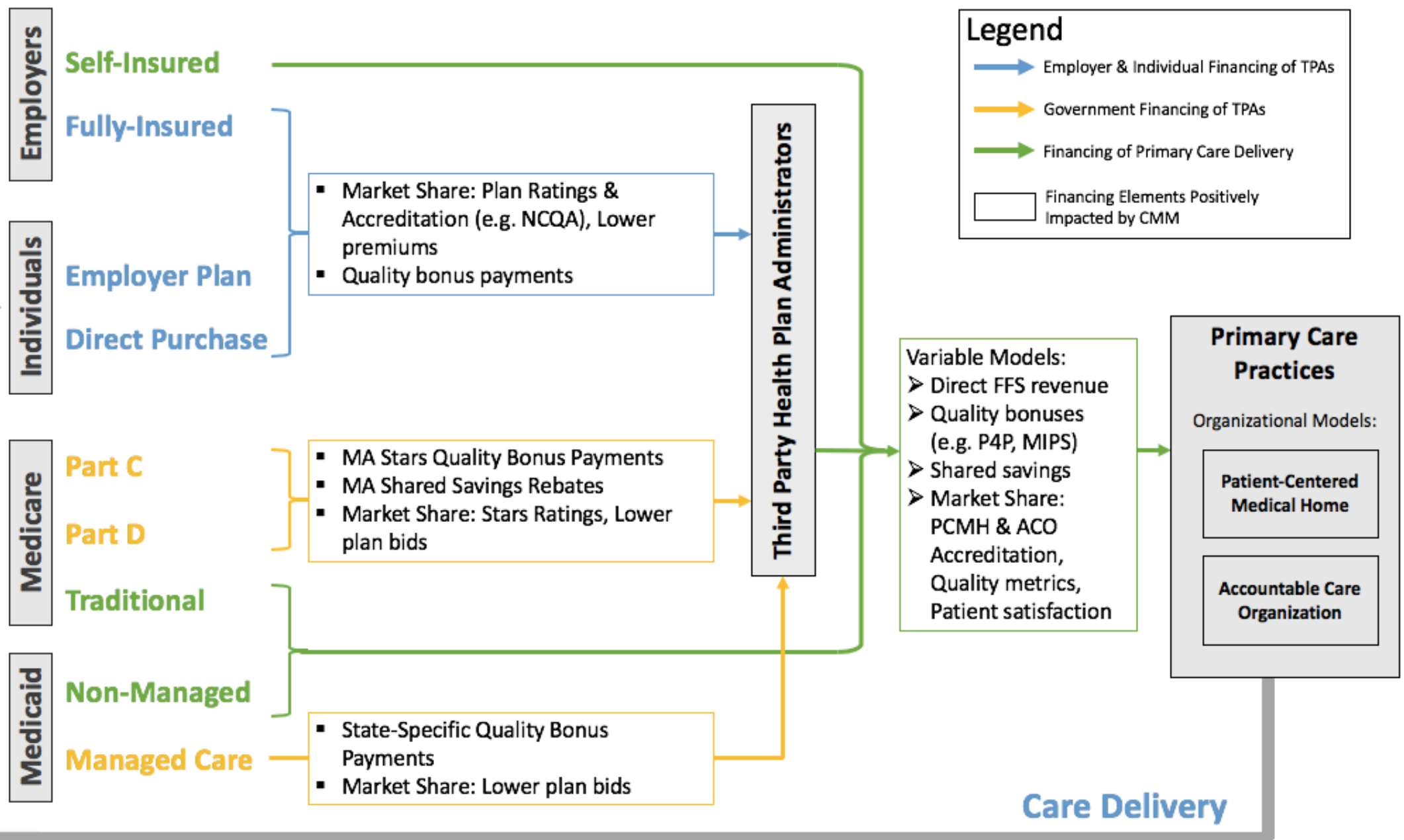

ACO = accountable care organization, $\mathrm{CPC}+=$ comprehensive primary care, $\mathrm{FFS}=$ fee for service, $\mathrm{MA}=$ Medicare Advantage, $\mathrm{MIPS}=$ Merit-Based Incentive Payment System, $\mathrm{NCQA}=$ National Committee for Quality Assurance, $\mathrm{P} 4 \mathrm{P}=$ pay for performance, $\mathrm{PCMH},=$ patient centered medical home, $\mathrm{TPA}=$ third party administrator. 
Table 1. 2018 Medicare STAR measures influenced by pharmacist CMM services.

\begin{tabular}{|l|l|}
\hline Annual flu vaccine & Medication Reconciliation Post-Discharge \\
\hline Care for Older Adults - Medication Review & Plan All-Cause Readmissions \\
\hline Osteoporosis Management in Women who had a Fracture & Medication Adherence for Diabetes Medications \\
\hline Diabetes Care - Blood Sugar Controlled & Medication Adherence for Hypertension (RAS antagonists) \\
\hline Controlling Blood Pressure & Medication Adherence for Cholesterol (Statins) \\
\hline Rheumatoid Arthritis Management & MTM Program Completion Rate for CMR \\
\hline Reducing the Risk of Falling & Getting Needed Prescription Drugs \\
\hline Improving Bladder Control & \\
\hline
\end{tabular}

Table 2. Billing codes related to CMM services.

\begin{tabular}{|l|l|l|}
\hline HCPCS Code & Code Description & $\begin{array}{l}\text { 2018 Reimbursement } \\
\text { Fee Schedule }\end{array}$ \\
\hline CPT 99211 & $\begin{array}{l}\text { Evaluation and management service office or } \\
\text { other outpatient visit that may not require the } \\
\text { presence of a physician }\end{array}$ & $\$ 22$ \\
\hline CPT 99490 & Chronic care management service - 20 minutes & $\$ 42$ \\
\hline CPT 99495 & Transitional care management - 14-day discharge & $\$ 167$ \\
\hline CPT 99456 & Transitional care management - 7-day discharge & $\$ 236$ \\
\hline G0438 & Annual Wellness Visit - initial visit & $\$ 175$ \\
\hline G0439 & Annual Wellness Visit - subsequent visit & $\$ 119$ \\
\hline
\end{tabular}

